\title{
One-year evolution of DLCO changes and respiratory symptoms in patients with post COVID-19 respiratory syndrome
}

\author{
Alberto Fortini ${ }^{1}$ - Aurelio Rosso ${ }^{1} \cdot$ Paolo Cecchini $^{2} \cdot$ Arianna Torrigiani $^{1} \cdot$ Aldo Lo Forte $^{1} \cdot$ Paolo Carrai $^{1}$. \\ Chiara Alessi $^{1} \cdot$ Francesca Fabbrizzi $^{1}$ - Elena Lovicu ${ }^{1} \cdot$ Serena Sbaragli ${ }^{1} \cdot$ Antonio Faraone $^{1}$
}

Received: 12 December 2021 / Accepted: 10 January 2022 / Published online: 21 January 2022

(c) The Author(s), under exclusive licence to Springer-Verlag GmbH Germany 2022

\begin{abstract}
Purpose During a follow-up program of patients admitted for COVID-19 at our non-ICU Unit, we found that $37 \%$ of them had decreased diffusing lung capacity for carbon monoxide (DLCO) 3-6 months after discharge. This prospective observational study aimed to evaluate the evolution of changes in DLCO and respiratory symptoms at the 1-year follow-up visit. Methods Seventeen (mean age 71 years; 8 males) of 19 eligible patients (DLCO $<80 \%$ of predicted at the 3-6 months follow-up visit) completed the 1-year follow-up visit. One patient refused to participate and 1 patient had died 3 months earlier from myocardial infarction. The visit included a self-reported structured questionnaire, physical exam, blood tests, ECG, and spirometry with DLCO.

Results Mean DLCO was significantly improved at the 1-year visit (from $64 \%$ of predicted at 3-6 months to $74 \%$ of predicted at 1 year; $P=0.003)$. A clinically significant increase in DLCO (10\% or greater) was observed in 11 patients $(65 \%)$ with complete normalization ( $>80 \%$ of predicted) in $6(35 \%)$; in the other $6(35 \%)$ it remained unchanged. The prevalence of exertional dyspnea $(65-35 \%, P=0.17)$, cough $(24-18 \%, P=1)$, and fatigue $(76-35 \%, P=0.04)$ decreased at the 1 -year visit. Conclusion These results suggest that DLCO and respiratory symptoms tend to normalize or improve 1 year after hospitalization for COVID-19 in most patients. However, there is also a non-negligible number of patients (about one-third) in whom respiratory changes persist and will need prolonged follow-up.
\end{abstract}

Keywords Post-acute sequelae of SARS-CoV-2 infection $\cdot$ Long-COVID $\cdot$ DLCO $\cdot$ Respiratory symptoms

\section{Introduction}

A high percentage of COVID-19 patients continue to complain of symptoms, particularly dyspnea and fatigue, in the months following hospital discharge [1-3]. Pulmonary functional alterations are frequently detected, in particular a reduction in lung diffusion capacity [1,2]. A follow-up program conducted 3-6 months after discharge from our nonICU COVID-19 ward showed that 19 of 51 patients (37.2\%) had mild or moderate changes in diffusing lung capacity for carbon monoxide (DLCO), associated with exertional dyspnea [2]. This prospective observational study aimed to

Alberto Fortini

xfortini@gmail.com; alberto.fortini@uslcentro.toscana.it

1 Internal Medicine, San Giovanni di Dio Hospital, Via di Torregalli 3, 50143 Florence, Italy

2 Pneumology, San Giovanni di Dio Hospital, Florence, Italy evaluate the 1-year evolution of DLCO changes and respiratory symptoms of these patients.

\section{Methods}

Patients discharged from our non-ICU COVID ward during the first wave of COVID-19 pandemic (from March to May 2020) were recalled for follow-up 3-6 months and 1 year later. The follow-up program was developed and carried out by a team of internal medicine physicians with the collaboration of two pulmonologists. It included anamnesis, a structured questionnaire on persisting symptoms, physical examination, and pneumological evaluation with pulmonary function tests (detailed description in the manuscript by Fortini et al. [2]). This prospective observational study included the patients who showed a reduction in DLCO ( $<80 \%$ of predicted) at the 3-6 month follow-up visit, and assessed the evolution of respiratory function and respiratory 
symptoms at the 1-year follow-up visit. The following spirometric parameters were considered: forced vital capacity (FVC), forced expiratory volume in one second (FEV1), FEV1/FVC, total lung capacity (TLC), and DLCO. A 10\% or greater intersession change of these parameters was considered clinically significant [4]. Among the self-reported complaints by the patient, we considered exertional dyspnea, cough and fatigue as respiratory symptoms. Fatigue was included among the respiratory symptoms because, despite being a multifaced phenomenon due to different mechanisms, the respiratory component is certainly very relevant [5].

\section{Statistical analysis}

GNU PSPP Statistical Analysis Software (https://www.gnu. org/software/pspp/) was used for statistical analyses. The data were presented as numbers and/or percentages for categorical variables and mean and standard deviation (SD) or median and interquartile range (IQR) when appropriate for continuous ones. The normality of data distribution was assessed using the Shapiro-Wilk test. Normally distributed continuous variables were compared with the Student's $t$ test, non-normally distributed variables with Mann-Whitney test. Categorical variables were compared with $\chi^{2}$ or Fisher exact test. $P<0.05$ was considered statistically significant.

This study was approved by the Institutional Review Board of the Department of Medicine of the "Azienda USL Toscana Centro", Firenze, Italy. Patients provided written informed consent to participate in the study.

\section{Results}

Seventeen of the 19 patients eligible for the study (with DLCO $<80 \%$ expected at the 3-6 month follow-up visit) completed the 1-year follow-up visit; 1 patient refused to participate and 1 patient had died 3 months earlier from myocardial infarction. Patients were followed up for a mean (SD) of 128 (18) days at 3-6 months, and 361 (25) at 12 months.

The mean (SD) age was 71 (11) years; 8 (47\%) were males and 9 (53\%) were females. Pulmonary function tests were completed in all patients. No patient underwent home oxygen therapy and no patient had undergone a specific respiratory rehabilitation program. No patient had a hospitalization between the 2 follow-up visits.

Table 1 shows the values of arterial blood gas analysis and spirometric examination at 3-6 months and 1 year after hospital discharge.

Mean DLCO was significantly improved at the 1 -year visit (from $64 \%$ of predicted at 3-6 months to $74 \%$ of predicted at 1 year; $P=0.003$ ). A clinically significant
Table 1 Results of arterial blood gas analysis and spirometry with DLCO at the 3-6 month and 1-year visit

\begin{tabular}{|c|c|c|c|}
\hline Patients $(n=17)$ & $3-6$ month visit & 1-year visit & $P$ \\
\hline \multicolumn{4}{|l|}{ Arterial blood gas } \\
\hline $\mathrm{Pa} 02 / \mathrm{Fi} 02$, mean (SD) & $444(38)$ & $422(33)$ & 0.74 \\
\hline $\mathrm{Pa} 02 / \mathrm{Fi} 02<350$, n. $(\%)$ & $1(6)$ & 0 & \\
\hline \multicolumn{4}{|l|}{ Spirometry } \\
\hline $\begin{array}{l}\text { FVC\% of predicted,, mean } \\
\text { (SD) }\end{array}$ & $92(19)$ & $95(22)$ & 0.46 \\
\hline $\mathrm{FVC}<80 \%, n(\%)$ & $4(24)$ & $4(24)$ & 1 \\
\hline $\begin{array}{l}\text { FEV1\% of predicted, mean } \\
\text { (SD) }\end{array}$ & $92(21)$ & $90(22)$ & 0.09 \\
\hline FEV1 < $80 \% n(\%)$ & $5(29)$ & $5(29)$ & 1 \\
\hline FEV1/FVC $\times 100$, mean $(\mathrm{SD})$ & $73(11)$ & $71(10)$ & 0.31 \\
\hline FEV1/FVC $<70 \% n(\%)$ & $4(24)$ & $5(29)$ & 1 \\
\hline $\begin{array}{l}\text { TLC, } \% \text { of predicted, mean } \\
\text { (SD) }\end{array}$ & $88(19)$ & $96(22)$ & 0.09 \\
\hline $\mathrm{TLC}<80 \% n(\%)$ & $6(35 \%)$ & $4(24)$ & 0.71 \\
\hline $\begin{array}{l}\text { DLCO, \% of predicted, mean } \\
\text { (SD) }\end{array}$ & $64(12)$ & $74(16)$ & 0.003 \\
\hline DLCO $<80 \%, n(\%)$ & $17(100)$ & $11(65 \%)$ & 0.018 \\
\hline
\end{tabular}

increase in DLCO (10\% or greater) was observed in 11 patients $(65 \%)$; in the other 6 patients (35\%) the DLCO was unchanged. DLCO at 1 year normalized ( $>80 \%$ of predicted) in 6 patients (35\%) (Fig. 1).

Table 2 shows some characteristics of patients in whom DLCO significantly improved at the 1-year visit and of patients in whom DLCO unchanged. Mean age and mean Charlson comorbidity index were approximately 3 years and 1 point lower in the first group than in the second group, respectively; however, the differences did not reach statistical significance.

Exertional dyspnea, cough and fatigue were self-reported by $11(65 \%), 4(24 \%)$ and $13(76 \%)$ patients at 3-6 months visit and by $6(35 \%), 3(18 \%)$ and $6(35 \%)$ patients at 1 -year visit, respectively. The prevalence of all of these symptoms decreased from the 3 to 6 month visit to the 1 year visit, but the difference was statistically significant only for persistent fatigue ( $P=0.04$ ) (Fig. 2). At least 1 of these 3 symptoms was reported by 11 patients (65\%), 2 symptoms by 6 patients (35\%); none of the patients reported all the 3 symptoms.

\section{Discussion}

In this study, we prospectively assessed the 1-year evolution of lung function and respiratory symptoms in 17 hospitalized non-intubated patients with COVID-19 pneumonia who had shown a significant clinical reduction of DLCO 3-6 months after hospital discharge. 
Fig. 1 DLCO at 3-6 months and 1-year after COVID-19 related hospitalization. Each colored line represents 1 patient. $D L C O$ diffusing lung capacity for carbon monoxide

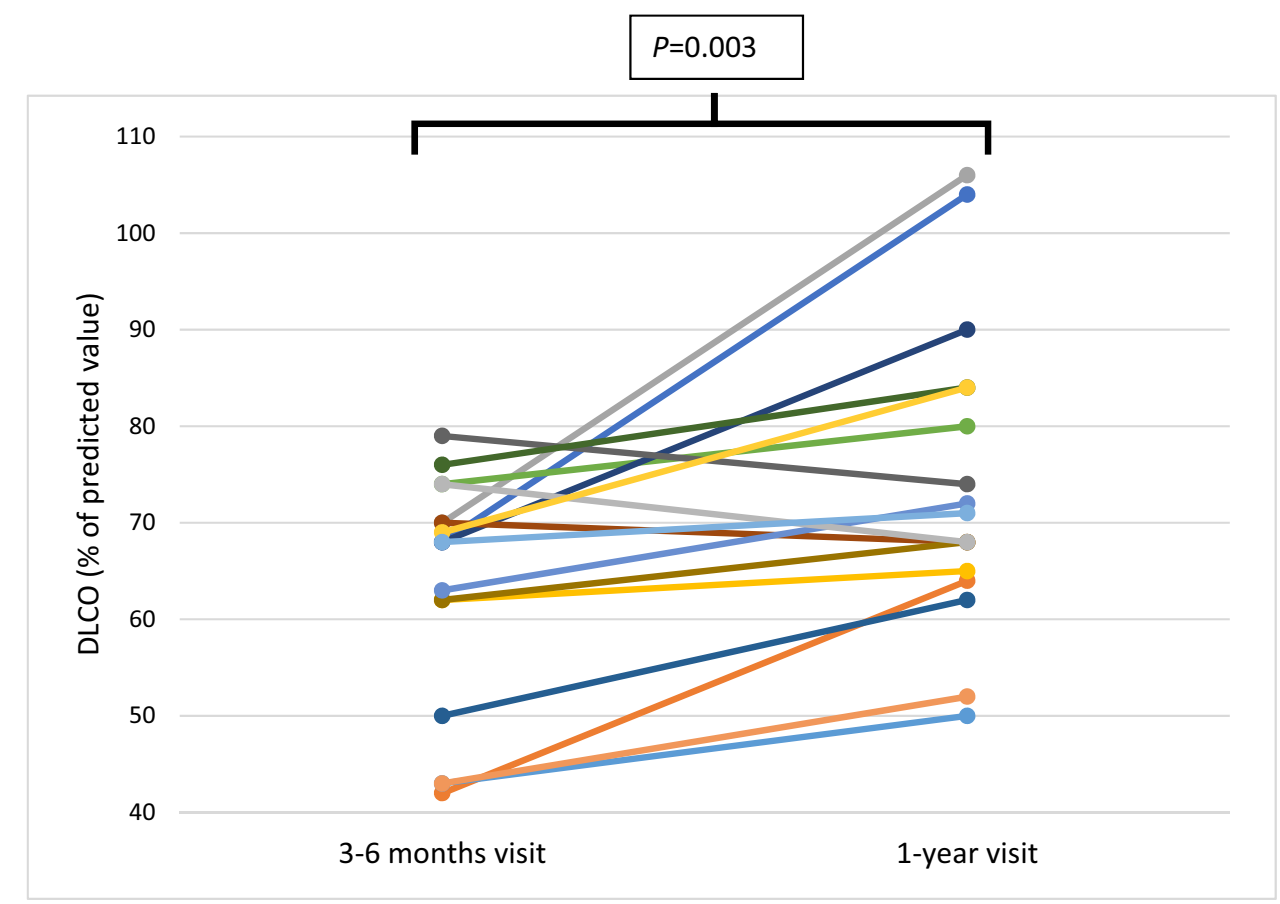

Table 2 Demographic and clinical characteristics of patients with improved or unchanged DLCO at the 1-year visit

\begin{tabular}{llll}
\hline & DLCO improved $(N=11)$ & DLCO unchanged $(N=6)$ & $P$ \\
\hline Male gender, $N(\%)$ & $5(45)$ & $3(50)$ & $73.8(11.2)$ \\
Age, years, mean (SD) & $70.2(10.9)$ & $4.5(2.9)$ & 0.26 \\
Charlson Comorbidity Index, mean (SD) & $3.6(2.2)$ & $2(33)$ & 1 \\
Non-invasive ventilation during hospitalization, $N(\%)$ & $3(27)$ & $17.3(8.8)$ & 0.25 \\
Length of hospital stay, days, mean (SD) & $20.2(12.2)$ & $4(67)$ & 0.69 \\
Persisting exertional dyspnea at 1-year, $N(\%)$ & $7(64)$ & $2(33)$ & 1 \\
Persisting cough at 1-year, $N(\%)$ & $1(9)$ & $2(33)$ & 0.51 \\
Persisting fatigue at 1-year, $N(\%)$ & $4(36)$ & $590(457 ; 923)$ \\
D-dimer, nanogr/ml, median (IQR)* & $453(292 ; 615)$ & $92(50 ; 119)$ \\
Ferritin, ng/ml, median (IQR)* & $55(32 ; 81)$ & $0.5(0.3 ; 0.7)$ \\
CRP, mg/dL, median (IQR)* & $0.24(0,1 ; 0.3)$ & 0.29 \\
\hline
\end{tabular}

$I Q R$ interquartile range. D-dimer reference range: $<500$ nanogr $/ \mathrm{ml}$. Ferritin reference range: $20-200 \mathrm{ng} / \mathrm{ml}$. CRPC-reactive protein; reference range: $<0.5 \mathrm{mg} / \mathrm{dL}$

*Values obtained at the 1-year visit

The main results indicate that in patients with COVID19 , changes in diffusing capacity of the lung and respiratory symptoms observed 3-6 months after hospitalization tend to improve at the 1-year follow-up visit in most patients, but not in all. Indeed, DLCO significantly improved in 65\% of patients, remained unchanged in $35 \%$, and did not significantly deteriorate in any of the patients. Patients with improved DLCO had on average a lower age and comorbidity index than the others. However, the differences did not reach statistical significance, probably due to the limited number of patients, and therefore we are unable to provide reliable indications. Overall, at the 1-year visit, DLCO normalized ( $>80 \%$ predicted) in $35 \%$ of the patients. In addition, the prevalence of respiratory symptoms decreased 1 year after hospital admission, but at least 1 symptom was still present in $65 \%$ of patients.

These results are substantially in agreement with the data currently available in the literature. Wu et al. [6] recently reported the results of a study that prospectively evaluated respiratory outcomes of 83 patients 3-6-9 and 12 months after hospitalization for COVID-19. Overall, they observed temporal improvement in lung physiology and exercise 
Fig. 2 Persisting respiratory symptoms at 3-6 months and 1 -year after COVID-19 related hospitalization

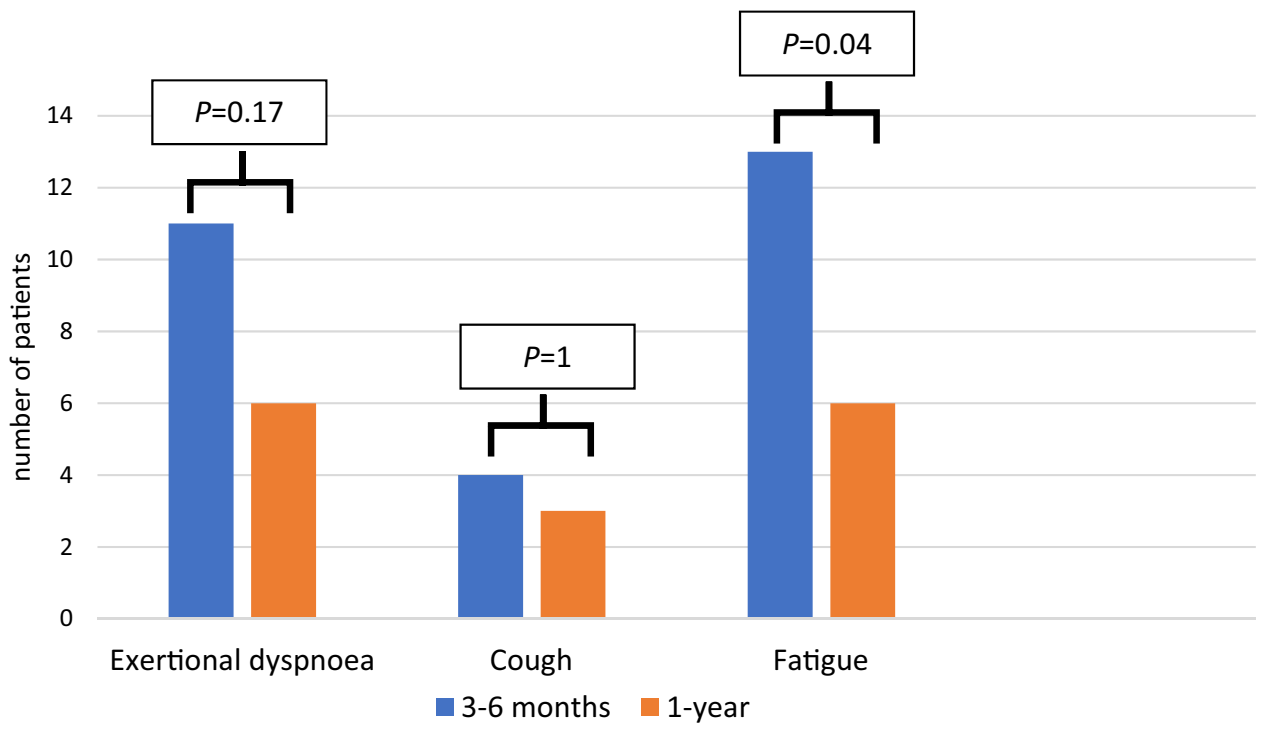

capacity in most patients. However, persistent decreased DLCO and persistent high-resolution computer tomography (HRCT) changes were still evident in approximately one-third and one-quarter of patients at the 12-month visit, respectively. The HRCT changes consisted mainly of ground-glass opacity, while none of the patients showed evidence of established fibrosis or progressive interstitial changes. In our program of follow-up visits, the systematic execution of the HRCT scan was not scheduled to avoid the patients to unnecessary radiation. However, in the 4 patients submitted to HRCT scan at the 1-year visit, we also found some ground-glass opacities without a clear picture of fibrosis. In a large observational Chinese study, the percentage of patients with at least one sequelae symptom decreased from $68 \%$ at 6 months to $49 \%$ at 12 months $(P<0.0001)$, but the proportion of patients with dyspnea slightly increased from $26 \%$ at 6 -month visit to $30 \%$ at 12 -month visit $(P=0.14)$ [7]. A prospective bicentric study showed a moderate reduction in the prevalence of fatigue (58-46\%) and shortness of breath (36-21\%) in 90 patients evaluated 90 and 365 days after hospitalization for COVID-19 [8].

A notable result of our study is that no patients showed further deterioration in respiratory function from 3 to 6 months to 1 year after admission. These data seem to suggest that changes in the pulmonary interstitium, frequently detected in the first months after the acute phase of COVID19 [9], do not tend to worsen over time. The possible evolution into pulmonary fibrosis secondary to COVID-19 is a matter of concern [10]. The data in the literature on the long-term progression of pulmonary interstitial changes are limited and currently inconclusive [10]. However, in a Chinese study, HRCT scans at 9 and 12 months after hospitalization for COVID-19 showed no evidence of established fibrosis and progressive interstitial changes [6]. In a 70-year-old patient with severe COVID-19 pneumonia requiring mechanical ventilation, a complete histopathological and functional recovery was observed 6 months later [11]. An indirect support to the no further evolution towards fibrosis of the pulmonary alterations detected in the first months post-COVID-19 is provided by the data on severe acute respiratory syndrome (SARS). A 15-year follow-up study involving 71 patients with SARS showed that interstitial anomalies and functional decline recovered in the first 2 years after infection and then remained stable [12].

This study has some limitations. First, it is a single-center study including a small number of patients. However, it should be considered that, unlike other studies with greater numbers, this prospective study was focused only on patients who had shown alterations in DLCO after 3-6 months of hospitalization for COVID-19. Second, patients were not systematically evaluated with radiological investigations, such as HRCT. We considered it appropriate not to include HRCT in the follow-up program to avoid patients being subjected to unnecessary radiation. Furthermore, we felt that clinical and functional assessments provided more relevant information for patient care than morphological ones.

\section{Conclusions}

The results of this study indicate that in most patients DCLO and symptoms related to respiratory dysfunction tend to normalize or improve 1 year after hospitalization for COVID-19. However, there is also a non-negligible number of patients in whom functional respiratory dysfunction and symptoms persist, and a prolonged follow-up will be required to understand the further clinical evolution. An encouraging finding is that no patient has experienced 
clinically significant worsening of DLCO and respiratory symptoms, suggesting that COVID-19-induced lung injury does not seem to evolve over time.

Since this study included a limited number of patients and was carried out in a single center, the data need to be confirmed by larger multicenter studies.

Author contributions All authors contributed to the study conception and design. Material preparation and data collection were performed by all authors. Data analysis was performed by AF, AR and AT. The first draft of the manuscript was written by AF, AT and AF. All authors read and approved the final manuscript.

Funding This research did not receive any specific grant from funding agencies in the public, commercial, or not-for-profit sectors.

Availability of data and material All study data are stored in an electronic database (Microsoft Excel spreadsheet, version 2013) and is available for review.

\section{Declarations}

Conflict of interest The authors declare that they have no conflicts of interest.

Ethics approval This study was approved by the Institutional Review Board of the Department of Medicine of the "Azienda USL Toscana Centro", Firenze, Italy. The authors declare that all procedures performed in this study are in accordance with ethical standards of the institutional and national research committee and with the 1964 Helsinki declaration and its later amendments or comparable ethical standards.

Consent to participate Patients provided written informed consent to participate in the study.

Consent for publication Patients signed informed consent regarding publishing their data.

\section{References}

1. Huang C, Huang L, Wang Y, et al. 6-month consequences of COVID-19 in patients discharged from hospital: a cohort study.
Lancet. 2021;397:220-32. https://doi.org/10.1016/S01406736(20)32656-8

2. Fortini A, Torrigiani A, Sbaragli S, et al. COVID-19: persistence of symptoms and lung alterations after 3-6 months from hospital discharge. Infection. 2021;49:1007-15. https://doi.org/10.1007/ s15010-021-01638-1.

3. Jiang DH, Roy DJ, Gu BJ, Hassett LC, McCoy RG. Postacute sequelae of severe acute respiratory syndrome coronavirus 2 infection: a state-of-the-art review. JACC Basic Transl Sci. 2021;6:796-811. https://doi.org/10.1016/j.jacbts.2021.07.002.

4. Pellegrino R, Viegi G, Brusasco V, et al. Interpretative strategies for lung function tests. Eur Respir J. 2005;26:948-68. https://doi. org/10.1183/09031936.05.00035205.

5. Gruet M. Fatigue in chronic respiratory diseases: theoretical framework and implications for real-life performance and rehabilitation. Front Physiol. 2018;9:1285. https://doi.org/10.3389/ fphys.2018.01285 (Published 2018 Sep 19).

6. Wu X, Liu X, Zhou Y, et al. 3-month, 6-month, 9-month, and 12-month respiratory outcomes in patients following COVID19-related hospitalisation: a prospective study. Lancet Respir Med. 2021;9:747-54. https://doi.org/10.1016/S2213-2600(21) 00174-0.

7. Huang L, Yao Q, Gu X, et al. 1-year outcomes in hospital survivors with COVID-19: a longitudinal cohort study. Lancet. 2021;398:747-58. https://doi.org/10.1016/S0140-6736(21) 01755-4.

8. Becker C, Beck K, Zumbrunn S, et al. Long COVID 1 year after hospitalisation for COVID-19: a prospective bicentric cohort study. Swiss Med Wkly. 2021;151:w30091. https://doi.org/10. 4414/smw.2021.w30091.

9. Vianello A, Guarnieri G, Braccioni F, et al. The pathogenesis, epidemiology and biomarkers of susceptibility of pulmonary fibrosis in COVID-19 survivors. Clin Chem Lab Med. 2021. https://doi. org/10.1515/cclm-2021-1021 (published online ahead of print, 2021 Nov 16).

10. Spagnolo $\mathrm{P}$, Balestro E, Aliberti S, et al. Pulmonary fibrosis secondary to COVID-19: a call to arms? Lancet Respir Med. 2020;8:750-2. https://doi.org/10.1016/S2213-2600(20)30222-8.

11. Boehm A, Luger AK, Schmitz K, et al. A spark of hope: histopathological and functional recovery after critical COVID-19. Infection. 2021. https://doi.org/10.1007/s15010-021-01678-7 (published online ahead of print, 2021 Aug 25).

12. Zhang P, Li J, Liu H, et al. Long-term bone and lung consequences associated with hospital-acquired severe acute respiratory syndrome: a 15-year follow-up from a prospective cohort study. Bone Res. 2020;8:8. https://doi.org/10.1038/s41413-020-0084-5 (published correction appears in Bone Res. 2020 Sep 21;8(1):34; Published 2020 Feb 14). 